$\underline{\underline{\text { IIIIIII }}} \stackrel{\text { Open Access Articles }}{\text { Open }}$

\title{
An Extended Group Additivity Method for Polycyclic Thermochemistry Estimation
}

The MIT Faculty has made this article openly available. Please share how this access benefits you. Your story matters.

Citation

Han, Kehang et al. "An Extended Group Additivity Method for Polycyclic Thermochemistry Estimation." International Journal of Chemical Kinetics 50, 4 (February 2018): 294-303 @ 2018 Wiley Periodicals, Inc

As Published

http://dx.doi.org/10.1002/kin.21158

Publisher

Wiley Blackwell

Version

Author's final manuscript

Citable link

http://hdl.handle.net/1721.1/114934

Terms of Use

Creative Commons Attribution-Noncommercial-Share Alike

Detailed Terms

http://creativecommons.org/licenses/by-nc-sa/4.0/ 


\title{
An Extended Group Additivity Method for Polycyclic Thermochemistry Estimation
}

\author{
Kehang Han ${ }^{\mathrm{a}}$, Adeel Jamal ${ }^{\mathrm{a}}$, Colin A. Grambow ${ }^{\mathrm{a}}$, Zachary J. Buras ${ }^{\mathrm{a}}$, William H. Green ${ }^{\mathrm{a}, *}$ \\ ${ }^{a}$ Department of Chemical Engineering, Massachusetts Institute of Technology, Cambridge, MA 02139, United States
}

\begin{abstract}
Automatic kinetic mechanism generation, virtual high-throughput screening, and automatic transition state search are currently trending applications requiring exploration of a large molecule space. Large scale search requires fast and accurate estimation of molecules' properties of interest, such as thermochemistry. Existing approaches are not satisfactory for large polycyclic molecules: considering the number of molecules being screened, quantum chemistry (even cheap DFT methods) can be computationally expensive, and group additivity, though fast, is not sufficiently accurate. This paper provides a fast and moderately accurate alternative by proposing a polycyclic thermochemistry estimation method that extends the group additivity method with two additional algorithms: similarity match and bicyclic decomposition. It significantly reduces $H_{f}(298 \mathrm{~K})$ estimation error from over 60 $\mathrm{kcal} / \mathrm{mol}$ (group additivity method) to around $5 \mathrm{kcal} / \mathrm{mol}, C_{p}(298 \mathrm{~K}$ ) error from $9 \mathrm{cal} / \mathrm{mol} / \mathrm{K}$ to 1 $\mathrm{cal} / \mathrm{mol} / \mathrm{K}$, and $S(298 \mathrm{~K})$ error from $70 \mathrm{cal} / \mathrm{mol} / \mathrm{K}$ to $7 \mathrm{cal} / \mathrm{mol} / \mathrm{K}$. This method also works well for heteroatomic polycyclics. A web application for estimating thermochemistry by this method is made available at http://rmg.mit.edu/molecule_search.
\end{abstract}

Keywords: polycyclics, thermochemistry, ring corrections

\section{Introduction}

Automatic kinetic mechanism generation [1], virtual high-throughput screening in drug discovery [2], and automatic transition state search [3] are a few examples of current trending applications where a large space of molecules is to be explored. For instance, RMG [1], an automatic kinetic mechanism generation package, typically scans $10,000 \sim 1,000,000$ of species for every single run. Such large scale of screening for which even cheap DFT methods are not affordable, requires fast estimation of molecular properties, such as thermochemistry. Group additivity method has been widely used since they were invented by Benson [4] as described by Eq. 1 (taking enthalpy as an example). Applications like RMG use it as a primary estimation method, benefiting from its convenience and interpretability.

\footnotetext{
* Corresponding author

Email address: whgreen@mit.edu (William H. Green)
} 


$$
H_{f}(298 K)=\sum_{i=1}^{N_{\text {atom }}} G A V_{i}
$$

where $G A V_{i}$ is group additivity value for $i^{\text {th }}$ atom centered group.

However, due to its underlying assumption that each atom-based group is independent and their contributions are additive, group additivity methods have difficulty estimating the thermochemistry of cyclic molecules, since ring strain is a joint effect among many ring atoms that is beyond singleatom-based scope.

To handle this problem, Benson [4] and others [5, 6] proposed ring corrections on top of the normal atom-based group additivity scheme (Eq. 2).

$$
H_{f}(298 K)=\sum_{i=1}^{N_{\text {atom }}} G A V_{i}+\sum_{j=1}^{N_{\text {ring cluster }}}(\text { ring correction })_{j}
$$

where $(\text { ring correction })_{j}$ is additional strain contributed by ring cluster $j$ as a whole.

Note a ring cluster may consist of several individual rings that share at least one atom with at least one other individual ring in the cluster. To make accurate predictions, Eq. 2 requires correction data for every ring cluster in each molecule.

Since each ring cluster structure has its specific ring correction, and there are an extremely large number of possible fused ring clusters, this group additivity method only gives accurate predictions for molecules whose ring structures have been studied in the past. Estimation accuracy drops significantly when dealing with molecules with ring cluster structures not included in the database.

The root problem is that one cannot list the infinite number of possible ring clusters and prepare all the ring corrections. Due to the difficulty in acquiring data from ab initio calculations or experimental measurements, less data is available for molecules with larger ring clusters than for those with smaller ones. However, as cluster size increases more possible structural variations exist, which worsens the situation for estimating large polycyclics.

Therefore, we divide the problem of accurately estimating the thermochemistry of a polycyclic into two sub-problems based on the size of the ring cluster (number of smallest rings in the cluster, using Fan's algorithm [7] of Smallest Set of Smallest Rings) in the molecule:

- small cyclics ( $\leq 2$-ring molecules) and

- large cyclics ( $\geq 3$-ring molecules)

For the former problem, we calculate and organize the available ring corrections into a functional group tree that can find similar matches for any new small cyclics. For the latter problem, we develop a bicyclic-decomposition model which estimates large polycyclic ring cluster corrections by 
decomposing them into smaller ones and adding up the contributions from the fragments. Overall, we managed to bring down group additivity thermo prediction error from over $60 \mathrm{kcal} / \mathrm{mol}$ in some cases (original group additivity method in cases where the ring cluster structure of interest had not been studied previously) to $5 \mathrm{kcal} / \mathrm{mol}$ for both small cyclics and large cyclics as judged using the dataset of Ramakrishnan, et al. [8].

In this paper, we discuss our similarity match approach in Section 3 and our bicyclic-decomposition approach in Section 4. Additionally, to power these algorithms, we organize and precalculate ring corrections for a list of frequently seen ring cluster structures, with more details in Section 2.

\section{Pre-calculation}

The precalculated list of hydrocarbon molecules covers molecules with mostly small ring cluster structures (1-ring and 2-ring clusters, see Supporting Information). Some example molecules are shown in Figure 1.

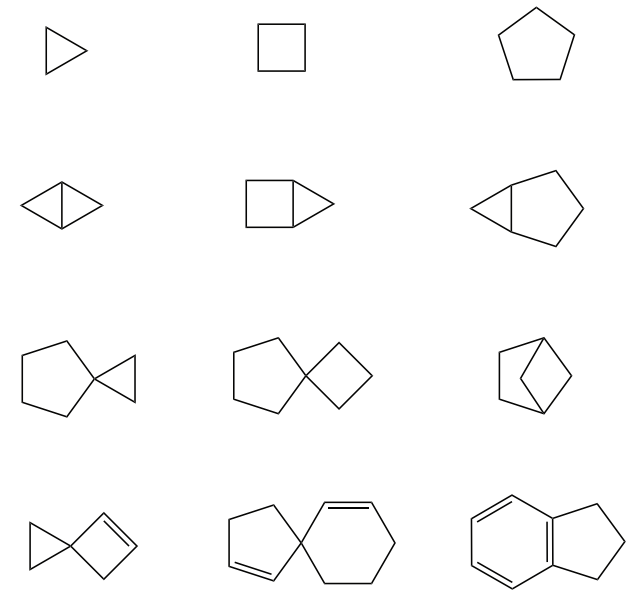

Figure 1: Example small cyclics in our database

To automate the data preparation process, a 3-step scheme was used as shown in Figure 2. Firstly, molecular identifiers are fed into RDKit Chem module [9] to generate initial XYZ coordinates. A GAUSSIAN job creator receives the molecular coordinates, composes GAUSSIAN 09 [10] job inputs and launches quantum chemistry jobs. To optimize the geometry and to compute vibrational frequencies at the optimized geometry $X Y Z_{\text {opt }}$, we used the DFT method at M06-2X/cc-pVTZ level of theory [11]. Once the quantum chemistry calculation finishes, RMG's Cantherm module [1] (additional cantherm information can be found at: http://reactionmechanismgenerator.github.io/RMG-Py/users/ 
cantherm/index.html) parses the output GAUSSIAN log file and calculates the thermochemical parameters such as $H_{f}(298 \mathrm{~K}), C_{p}$ and $S(298 \mathrm{~K})$ using the Rigid Rotor Harmonic Oscillator (RRHO) approximation. At each step, molecular representations (SMILES, XYZ, and optimized XYZ coordinates) are converted into RMG species objects and RMG's isomorphism check ensures that they still represent the same molecule.

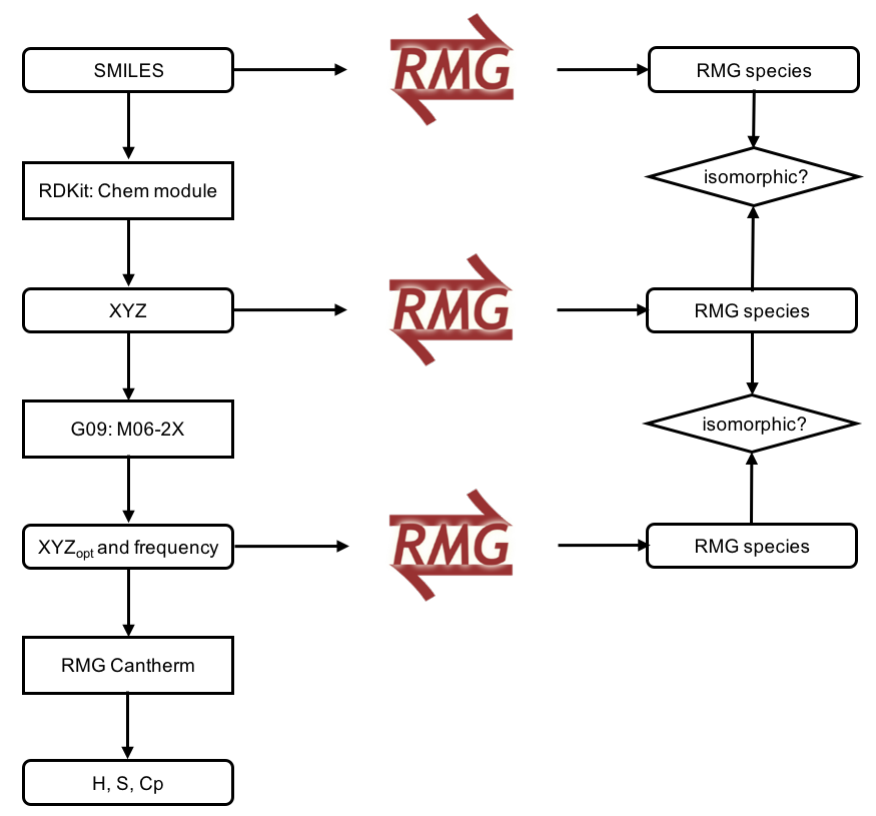

Figure 2: Quantum calculation scheme for small cyclic thermochemistry

Note that the single structure RRHO approach employed here only considers one conformer, and ignores anharmonicity, so it is expected to underestimate $S$ and $C_{p}$ for floppy rings.

\section{Similarity Match}

As discussed in the previous section, Eq. 2 needs exact matches of target ring clusters (otherwise no correction is applied at all), and thus requires extensive data to ensure high prediction accuracy.

Here we propose a similarity match algorithm that can find a similar ring with similar thermochemistry for situations where no exact matches are available.

\subsection{Cyclic trees}

The similarity algorithm greatly relies on data organization. The ring structures are organized into trees (we have two trees so far: monocyclic tree and polycyclic tree), see a sub-tree example in Figure 3. Nodes further down the tree have more specific structural details. The top layer defines the skeleton frame, for instance, s1_3_6 represents a bicyclic consisting of 3-member ring and 6-member ring with 1 
atom shared, and the next layer defines categories such as alkane, alkene, diene or aromatics. Finally, the bottom layer lists the most specific ring structures.

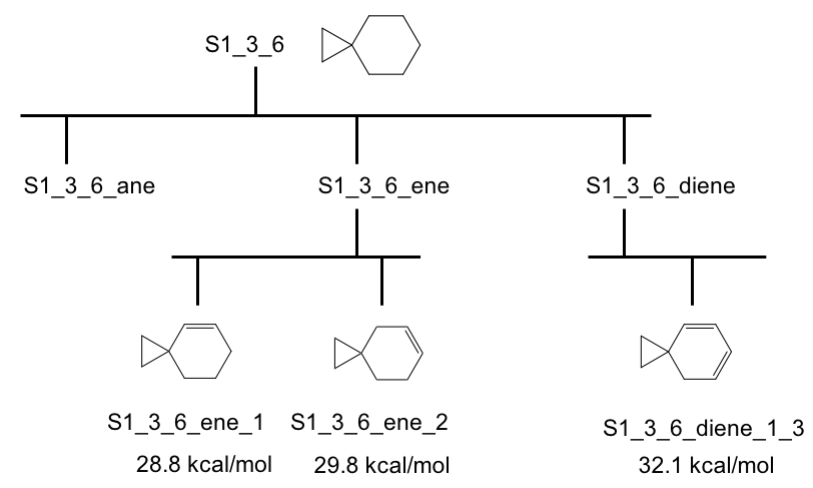

Figure 3: Example sub-tree that organizes polycyclic ring corrections with derived ring correction for enthalpy of formation

With this design, if a new molecule does not exactly match a known ring cluster, it can be classified as similar to some other nodes in the tree, and assigned the average of their values. For instance the molecule in Figure 4 most closely matches the second-layer node s1_3_6_diene in Figure 3. Since there is no exact match, the algorithm will use the average of the ring corrections of the children of s1_3_6_diene as its estimated correction.

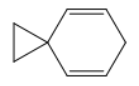

Figure 4: Example molecule which does not exactly match any node in the tree in Figure 3 . The tree gives enthalpy estimation of $32.1 \mathrm{kcal} / \mathrm{mol}$, while its real enthalpy of formation from quantum calculation in this study is $27 \mathrm{kcal} / \mathrm{mol}$, leading to around $5 \mathrm{kcal} / \mathrm{mol}$ error

\subsection{Model test}

To evaluate the performance of this similarity match algorithm, an external large quantum calculation dataset [8] is used. This dataset contains 134,000 molecules and has enabled several interesting big data studies $[12,13]$ that connect machine learning models to molecular property estimation. This paper selects cyclic molecules in that dataset as the test dataset and categorizes the cyclics into small cyclics (1-ring and 2-ring molecules, see example in Figure 5(a)), large linear cyclics (at least 3-ring molecules, and atoms are at most shared by two rings, see example in Figure 5(b)) and large fused cyclics (at least 3-ring molecules, rings are heavily fused (having atoms shared by at least 3 rings), see example in Figure 5(c)).

With the polycyclic tree (small cyclics calculated by the authors of this paper), the similarity match algorithm can successfully reduce the mean absolute error of $H_{f}(298 \mathrm{~K})$ from $32 \mathrm{kcal} / \mathrm{mol}$ 


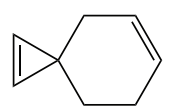

(a)

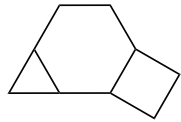

(b)

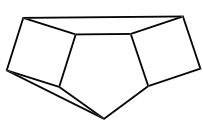

(c)

Figure 5: Example cyclics in each category: (a) small cyclics, (b) large linear cyclics, and (c) large fused cyclics

(original group additivity method in cases where the ring cluster structure of interest had not been studied previously) to $3 \mathrm{kcal} / \mathrm{mol}$ for small cyclics in the test dataset by Ramakrishnan, et al. [8] (see Table 1 and Figure 6), which is expected since the tree includes many pre-calculated small cyclic corrections.

Table 1: Mean absolute error $(\mathrm{kcal} / \mathrm{mol})$ of $H_{f}(298 \mathrm{~K})$ for each category in validataion dataset

\begin{tabular}{cccc}
\hline Method & small cyclics & large linear cyclics & large fused cyclics \\
\hline Group Additivity Method & 32 & 65 & 80 \\
+ Similarity Match & 3 & 29 & 40 \\
+ Bicyclic Decomposition & 3 & 4.9 & 9.8 \\
\hline
\end{tabular}

Even though there are no large polycyclics in the tree, this similarity match approach also improves the predictions for large polycyclics by sub-molecule isomorphism (see an example in the Supporting Information for how a 3-ring molecule matches a 2-ring node if no 3-ring node available in tree), cutting the mean absolute error by about a factor of two (Table 1).

To further improve the accuracy of large cyclics thermochemistry prediction, obvious approaches would require pre-calculated data on large cyclics. However, the number of possible large polycyclics increases rapidly with the number of rings. To avoid this poor scaling, we built a model that estimates ring corrections of polycyclics from known corrections for bicyclics, as discussed in Section 4.

\section{Bicyclic Decomposition}

\subsection{Method development}

Having available ring correction data mostly for small cyclics, a model that estimates the thermochemistry of large cyclics from small cyclic building blocks is needed. We tried three methods, as shown in Figure 7.

Method (a) simply sums up ring correction contributions from single rings that make up the targeted large cyclics. One main drawback of this method is that it only counts the ring strain 


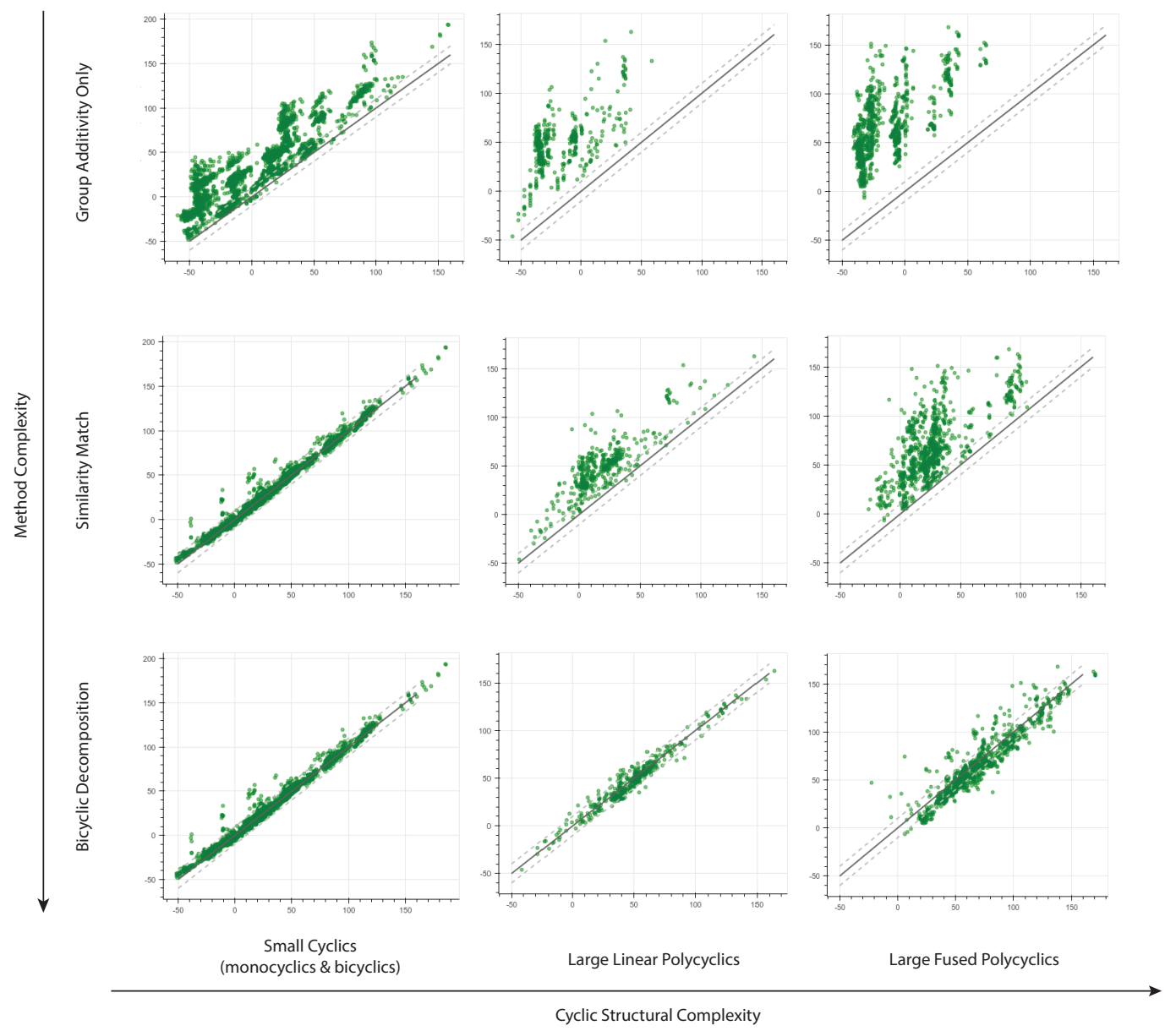

Figure 6: Predictions of enthalpy of formation (x axis) vs. quantum mechanics values (y axis, calculated by Ramakrishnan [8] using DFT method B3LYP/6-31G(2df,p)) with various models and cyclic types, unit: $\mathrm{kcal} / \mathrm{mol}$

contributions from individual rings and overlooks the extra strain from the fused part of the bicyclic ring $\mathrm{AB}$ in Figure 8. That is the reason method (a) underestimates the ring corrections by over 60 $\mathrm{kcal} / \mathrm{mol}$ in some cases (Figure 7). Significant discrepancy is observed between actual ring corrections needed to accurately estimate bicyclics and the sums of individual ring strain corrections (Figure 9).

Method (b) in Figure 7 divides a large cyclic into bicyclic components (called bicyclic decomposition), which automatically captures thermo contributions from fused parts. For instance, it decomposes a tricyclic into two bicyclics and estimates its ring corrections by taking the sum of bicylic corrections.

Method (b) reduces the error in predicted enthalpy of formation of tricyclo-octane to $27 \mathrm{kcal} / \mathrm{mol}$ by adding ring strain contributions from two fused parts in the tricyclic (Figure 7). However, it always 


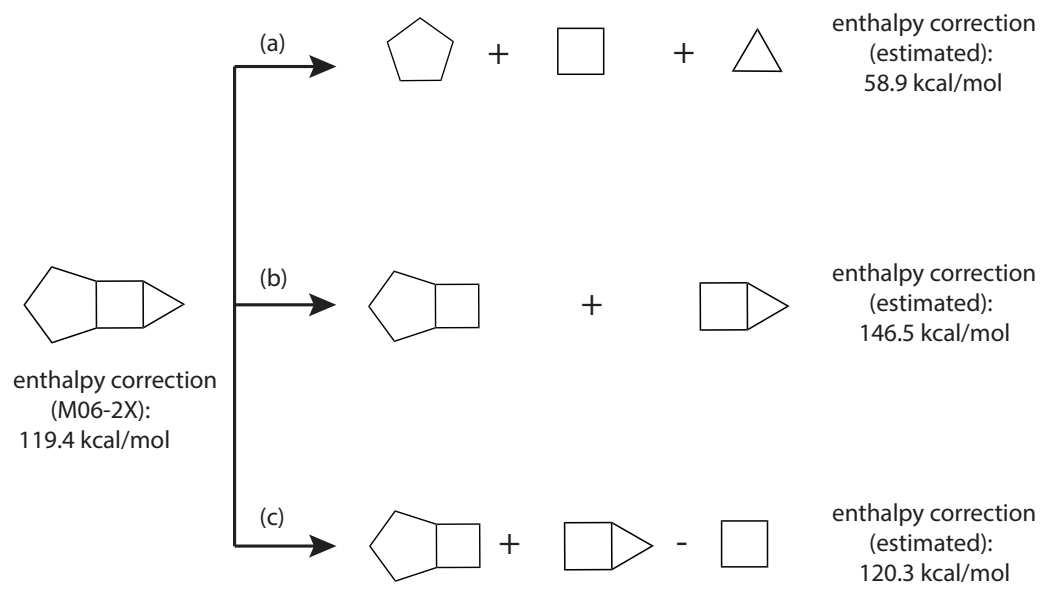

Figure 7: Large cyclic corrections estimation method evolution: (a) sum of individual single ring corrections, (b) sum of bicyclic corrections, and (c) sum of bicyclic corrections with overlapping ring correction subtraction

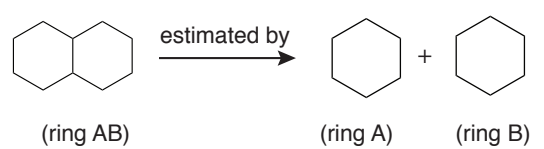

Figure 8: bicyclic AB correction estimated as sum of single ring A and B's corrections

over-predicts the enthalpy corrections, due to the fact that method (b) double counts the contribution of the middle 4-member ring. By eliminating the overlapped ring correction, method (c) shown in Figure 7 calculates a ring strain that agrees well with "true" ring correction (here we use our M06-2X calculations as "true" values). In this particular example, the prediction error is remarkably reduced to $0.9 \mathrm{kcal} / \mathrm{mol}$ by adopting the bicyclic decomposition approach. In Subsection 4.3, we conducted a more thorough test of the performance of method (c) .

\subsection{Bicyclic Correction Estimation}

We attempted to calculate commonly seen bicyclics and store them in the database. But polycylics may have bicyclic components that are not registered in our database. Often these are highly strained (e.g., consecutive double bonds in a ring) such as the examples in Figure 10.

To maintain high accuracy, adding relevant bicyclic clusters into the database would be a long term solution. In this study we developed an additional layer (Figure 11) in the original bicyclic decomposition method for cases where requested bicyclic components are not available or matched nodes are not similar enough to the target bicyclics. 


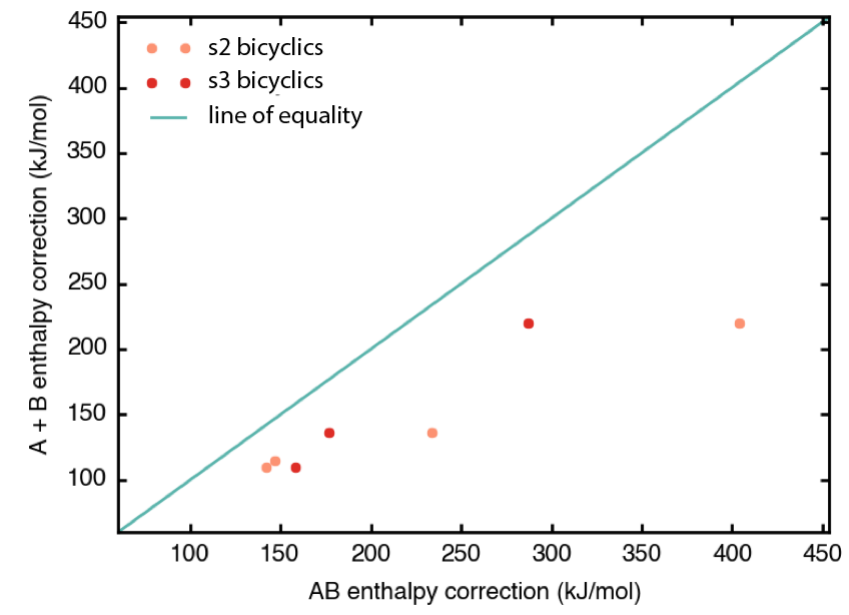

Figure 9: Ring corrections from bicyclics are very different from sum of corrections of individual single rings that make up the bicyclics. Note s2 bicyclics are those with 2-atom bridges, and s3 bicyclics are those with 3-atom bridges.

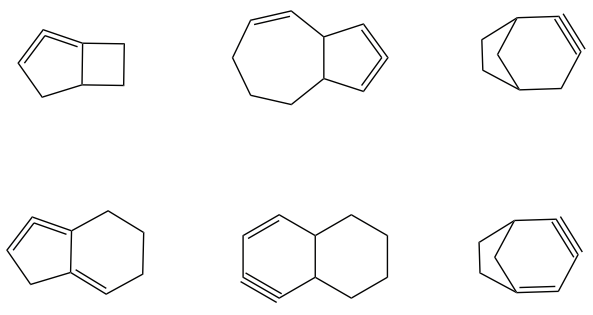

Figure 10: Bicyclic structures like these with high ring strain are usually not recorded in databases, but they can be formed as intermediates during reaction network exploration

\subsection{Model test}

Using the same polycylic dataset, the bicyclic decomposition method significantly reduces prediction error for both small and large cyclics (Figure 6).

For small cyclics ( $\leq 2$-ring molecules), bicyclics decomposition automatically falls back to the similarity match method, which guarantees the prediction accuracy achieved by similarity match, see Table 1. For large polycyclics, bicyclic decomposition outperforms similarity match, bringing the error down to $4.9 \mathrm{kcal} / \mathrm{mol}$ and $9.8 \mathrm{kcal} / \mathrm{mol}$ for large linear cyclics and large fused cyclics, respectively.

Our algorithm was aslo tested against the data set by Osmont, et al. [14] who used B3LYP/6$31 \mathrm{~g}(\mathrm{~d}, \mathrm{p})$ to calculate enthalpy of formation for propellanes. Our bicyclic decomposition algorithm, without running any further quantum chemistry calculations, was able to get enthalpies of dispiro[2.0.2.1] heptane, trispiro[2.0.2.0.2.0]nonane, trispiro[2.0.0.2.1.1] nonane and tetraspiro[2.0.0.0.2.1.1.1] undecane with DFT accuracy, as shown in Table 2. 


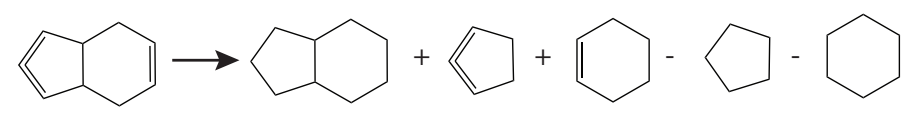

Figure 11: Bicyclic correction estimation scheme for bicyclics missing from the database

Table 2: Experimental and calculated enthalpy of formation at $298 \mathrm{~K}(\mathrm{kcal} / \mathrm{mol})$ for spiropentane related polycyclic compounds

\begin{tabular}{|c|c|c|c|c|}
\hline Structure & Name & Experimental value & Osmont [14] DFT data & This work \\
\hline & spiropentane & $44.3 \pm 0.2$ & 39.0 & 44.3 \\
\hline & dispiro[2.0.2.1]heptane & $72.4 \pm 0.8$ & 67.7 & 69.0 \\
\hline & trispiro[2.0.0.2.1.1]nonane & 102.7 & 96.5 & 100.1 \\
\hline & trispiro[2.0.2.0.2.0]nonane & $101.2 \pm 1.2$ & 96.5 & 97.1 \\
\hline & tetraspiro[2.0.0.0.2.1.1.1] undecane & $130.0 \pm 1.6$ & 125.3 & 131.3 \\
\hline
\end{tabular}

For users that are interested in using this method to estimate polycylic thermochemistry, a web application (http://rmg.mit.edu/molecule_search) is made available to allow users to input molecules (with elements of $\mathrm{C}, \mathrm{H}, \mathrm{O}$ ) using species identifiers such as SMILES, InChI, CAS number or species name and to compute that molecule's thermochemistry. A screenshot illustrating the output from this web tool is shown in Figure 12.

\section{Discussion}

The new thermochemistry estimator based on group addivity, which combines similarity match and the bicyclic decomposition method, predicts polycyclic thermochemistry more accurately. This extension makes the group additivity method generalizable for polycyclics without requiring much pre-calculated data.

\subsection{Large fused cyclics}

For bicyclics and linear polycyclics, the typical error of $3 \sim 5 \mathrm{kcal} / \mathrm{mol}$ is generally acceptable as a first approximation, especially when dealing with molecules with more than 10 carbons. The underlying reason that such a simple model performs well is that the decomposed bicyclic components act relatively independently; the thermodynamic contribution from the inter-bicyclic interaction is small compared with the ring strain contributions from the bicyclic itself. 


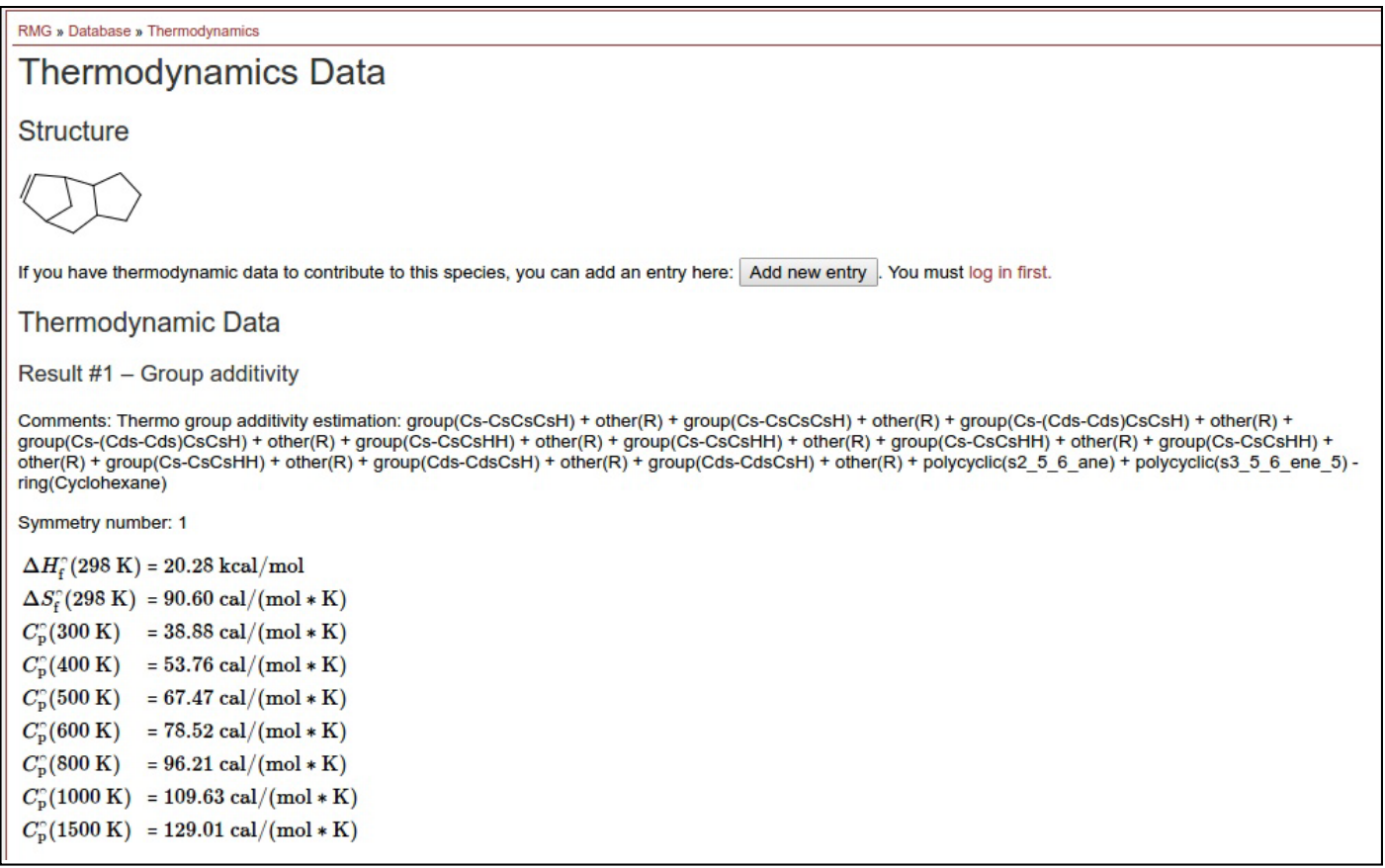

Figure 12: Example polycyclic thermochemistry estimation in web application at rmg.mit.edu

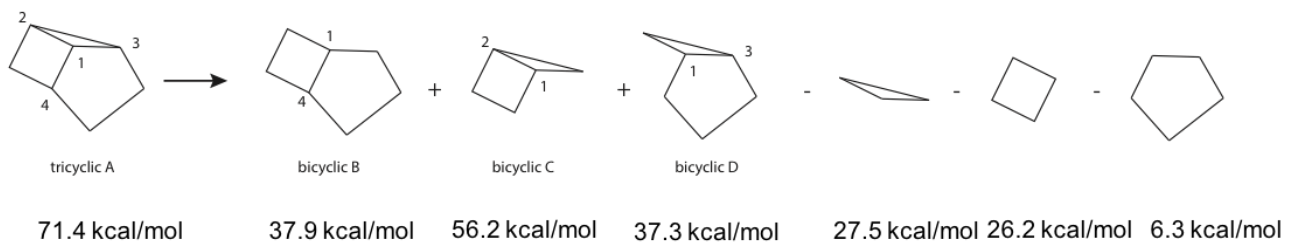

Figure 13: Bicyclic decompostion of an example heavily fused cyclic. The algorithm estimatates its ring correction for enthalpy of formation to be $71.4 \mathrm{kcal} / \mathrm{mol}$ using data from small rings, while direct quantum calculation gives 65 $\mathrm{kcal} / \mathrm{mol}$, leading to around $6 \mathrm{kcal} / \mathrm{mol}$ error

For heavily fused cyclics, there are atoms shared by more than two rings. For instance, tricyclic A in Figure 13 has one such atom (atom 1). To account for all the fused parts (bond 1-2, 1-3 and 1-4), the bicyclic decompostion algorithm has to decompose tricyclic A into 3 bicyclics; bicyclic B is for contribution from bond 1-4, bicyclic C for 1-2 and bicyclic D for 1-3. In this case, the thermodynamic contribution from the inter-bicyclic interaction is more important than in the large linear cyclic cases. That explains why the prediction error increases to $10 \mathrm{kcal} / \mathrm{mol}$ for those heavily fused cyclics.

\subsection{Hetero-atom polycyclics}

In many real applications, hetero-atoms such as $\mathrm{O}, \mathrm{S}$, and $\mathrm{N}$ are embedded in polycylics. There are usually fewer data available on heteropolycyclics than on polycyclic hydrocarbons. However if ring corrections depend more on ring structures than atom types, one can use the same database created 
by this paper to estimate heteroatom polycyclic thermochemistry.

Predictions made in this way for oxygen-embedded polycyclics agree well with the quantum mechanically calculated values for over 18,000 oxygen-embedded polycyclics [8] (Table 3). Predictions for hetero-atom polycyclics achieve similar accuracy to hydrocarbon polycyclics.

Table 3: Mean absolute error $(\mathrm{kcal} / \mathrm{mol})$ of $H_{f}(298 \mathrm{~K})$ for each category of oxygen-embeded polycyclics

\begin{tabular}{cccc}
\hline Method & small cyclics & large linear cyclics & large fused cyclics \\
\hline Group Additivity Method & 44 & 78 & 84 \\
+ Similarity Match & 5 & 34 & 40 \\
+ Bicyclic Decomposition & 5 & 6.6 & 10.6 \\
\hline
\end{tabular}

\subsection{Heat capacity and standard entropy predictions}

Table 4: Mean absolute error $(\mathrm{cal} / \mathrm{mol} / \mathrm{K})$ of $C_{p}(298 \mathrm{~K})$ for each category of polycyclics

\begin{tabular}{cccc}
\hline Method & small cyclics & large linear cyclics & large fused cyclics \\
\hline Group Additivity Method & 6.1 & 10.0 & 10.5 \\
+ Similarity Match & 1.1 & 2.0 & 2.7 \\
+ Bicyclic Decomposition & 1.1 & 0.7 & 1.7 \\
\hline
\end{tabular}

Besides $H_{f}(298 \mathrm{~K})$, the methods proposed by this paper also improve heat capacity prediction accuracy by a factor of $6 \sim 10$ for all three categories of polycyclics (Table 4 ). This can be crucial for chemical systems operated at temperatures other than $298 \mathrm{~K}$. For standard entropy $S(298 \mathrm{~K})$ predictions (Table 5), we also observed a similar accuracy boost using the bicyclic decomposition method. The good agreement seems to suggest the contributions to entropy and heat capacity from ring strains are also additive (although we note the entropy prediction for large fused cyclics has large uncertainties).

Table 5: Mean absolute error (cal/mol/K) of $S(298 \mathrm{~K})$ for each category of polycyclics

\begin{tabular}{cccc}
\hline Method & small cyclics & large linear cyclics & large fused cyclics \\
\hline Group Additivity Method & 44.5 & 93.3 & 103.5 \\
+ Similarity Match & 3.6 & 36.7 & 47.6 \\
+ Bicyclic Decomposition & 3.6 & 4.9 & 11.9 \\
\hline
\end{tabular}




\section{Conclusion}

The similarity match algorithm combined with the bicyclic decomposition model can estimate unknown ring corrections and can be applied to various kinds of polycyclics. By assuming bicyclic ring strain contributions are independent and additive, the proposed method is both interpretable and effective (mean absolute error of $H_{f}(298 \mathrm{~K}): 3 \sim 5 \mathrm{kcal} / \mathrm{mol}$ ) for bicyclics and linear polycyclics. It also achieves moderate accuracy (mean absolute error of $H_{f}(298 \mathrm{~K}): 10 \mathrm{kcal} / \mathrm{mol}$ ) for large heavily fused cyclics using the test dataset [8]. The method also shows good performance in heat capacity and entropy predictions. To further improve prediction accuracy, one might consider adding more terms that describe interactions between decomposed bicyclics. In addition, this method also accurately estimates the thermochemistry of some heteroatomic polycyclics (tested on oxygen-embedded polycyclics).

This proposed method provides a quick and moderately accurate way of estimating themochemistry of large unknown polycyclics where quantum mechanical calculation may be significantly more expensive. We recommend using it in applications where a large molecular space has to be scanned/explored with limited time such as during automatic mechanism generation, drug discovery, automatic transition state search, etc. We have made software implementing this new method freely available at http://rmg.mit.edu/molecule_search.

\section{Acknowledgments}

We gratefully acknowledge financial support from the DOE Gas Phase Chemical Physics program, grant No. DE-SC0014901. This research used resources of the National Energy Research Scientific (NERSC) Computing Center, a DOE Office of Science User Facility supported by the Office of Science of the U.S. Department of Energy under Contract No. DE-AC02-05CH11231.

\section{Supporting Information Available}

An example of querying a big cyclic molecule using similarity match algorithm and a collection of 190 computed thermochemistry of bicyclics are provided.

\section{References}

[1] C. W. Gao, J. W. Allen, W. H. Green, R. H. West, Reaction mechanism generator: Automatic construction of chemical kinetic mechanisms, Computer Physics Communications 203 (2016) 212 225. doi:10.1016/j.cpc.2016.02.013.

URL http://www.sciencedirect.com/science/article/pii/S0010465516300285 
[2] E. Gawehn, J. A. Hiss, G. Schneider, Deep Learning in Drug Discovery, Molecular Informatics 35 (1) (2016) 3-14. doi:10.1002/minf.201501008.

URL http://onlinelibrary.wiley.com/doi/10.1002/minf.201501008/abstract

[3] Y. V. Suleimanov, W. H. Green, Automated Discovery of Elementary Chemical Reaction Steps Using Freezing String and Berny Optimization Methods, Journal of Chemical Theory and Computation 11 (9) (2015) 4248-4259. doi:10.1021/acs.jctc.5b00407.

URL http://dx.doi.org/10.1021/acs.jctc.5b00407

[4] S. W. Benson, F. R. Cruickshank, D. M. Golden, G. R. Haugen, H. E. ONeal, A. S. Rodgers, R. Shaw, R. Walsh, Additivity Rules for the Estimation of Thermochemical Properties, Chem. Rev. 69 (1969) 279-324.

URL http://kinetics.nist.gov/kinetics/Detail?id=1969BEN/CRU279-324:0

[5] E. R. Ritter, J. W. Bozzelli, THERM: Thermodynamic property estimation for gas phase radicals and molecules, International Journal of Chemical Kinetics 23 (9) (1991) 767-778. doi:10.1002/ kin. 550230903.

URL http://onlinelibrary.wiley.com/doi/10.1002/kin.550230903/abstract

[6] T. H. Lay, T. Yamada, P.-L. Tsai, J. W. Bozzelli, Thermodynamic Parameters and Group Additivity Ring Corrections for Three- to Six-Membered Oxygen Heterocyclic Hydrocarbons, The Journal of Physical Chemistry A 101 (13) (1997) 2471-2477. doi:10.1021/jp9629497.

URL http://dx.doi.org/10.1021/jp9629497

[7] B. T. Fan, A. Panaye, J. P. Doucet, A. Barbu, Ring perception. A new algorithm for directly finding the smallest set of smallest rings from a connection table, Journal of Chemical Information and Computer Sciences 33 (5) (1993) 657-662. doi:10.1021/ci00015a002.

URL http://dx.doi.org/10.1021/ci00015a002

[8] R. Ramakrishnan, P. O. Dral, M. Rupp, O. A. v. Lilienfeld, Quantum chemistry structures and properties of 134 kilo molecules, Scientific Data 1 (2014) 140022. doi:10.1038/sdata.2014.22. URL http://www.nature.com/articles/sdata201422

[9] G. Landrum, et al., Rdkit: Open-source cheminformatics. URL http://www.rdkit.org

[10] M. J. Frisch, G. W. Trucks, H. B. Schlegel, G. E. Scuseria, M. A. Rob, J. R. Cheeseman, et al., Gaussian 09 Revision C.01, Gaussian Inc. Wallingford CT 2016. 
[11] Y. Zhao, D. G. Truhlar, The M06 suite of density functionals for main group thermochemistry, thermochemical kinetics, noncovalent interactions, excited states, and transition elements: two new functionals and systematic testing of four M06-class functionals and 12 other functionals, Theoretical Chemistry Accounts 120 (1) (2008) 215-241. doi:10.1007/s00214-007-0310-x. URL https://link.springer.com/article/10.1007/s00214-007-0310-x

[12] M. Rupp, A. Tkatchenko, K.-R. Muller, O. A. von Lilienfeld, Fast and accurate modeling of molecular atomization energies with machine learning, Physical Review Letters 108 (5) (2012) 058301. doi:10.1103/PhysRevLett.108.058301.

URL http://link.aps.org/doi/10.1103/PhysRevLett.108.058301

[13] R. Ramakrishnan, P. O. Dral, M. Rupp, O. A. von Lilienfeld, Big data meets quantum chemistry approximations: The machine learning approach, Journal of Chemical Theory and Computation 11 (5) (2015) 2087-2096. doi:10.1021/acs.jctc.5b00099.

URL http://dx.doi.org/10.1021/acs.jctc.5b00099

[14] A. Osmont, L. Catoire, I. Gkalp, Physicochemical Properties and Thermochemistry of Propellanes, Energy \& Fuels 22 (4) (2008) 2241-2257. doi:10.1021/ef8000423.

URL http://dx.doi.org/10.1021/ef8000423 